\title{
Non-linear wave-induced transient response of soil around a trenched pipeline
}

\author{
Fu-Ping Gao*, Ying-Xiang Wu \\ Institute of Mechanics, Chinese Academy of Sciences, Beijing 100080, People's Republic of China
}

Received 23 November 2004; accepted 11 May 2005

Available online 11 August 2005

\begin{abstract}
Submarine pipelines are always trenched within a seabed for reducing wave loads and thereby enhancing their stability. Based on Biot's poroelastic theory, a two-dimensional finite element model is developed to investigate non-linear wave-induced responses of soil around a trenched pipeline, which is verified with the flume test results by Sudhan et al. [Sudhan, C.M., Sundar, V., Rao, S.N., 2002. Wave induced forces around buried pipeline. Ocean Engineering, 29, 533-544] and Turcotte et al. [Turcotte, B.R., Liu, P.L.F., Kulhawy, F.H., 1984. Laboratory evaluation of wave tank parameters for wave-sediment interaction. Joseph H. Defree Hydraulic Laboratory Report 84-1, School of Civil and Environmental Engineering, Cornell University]. Non-linear wave-induced transient pore pressure around pipeline at various phases of wave loading is examined firstly. Unlike most previous investigations, in which only a single sediment layer and linear wave loading were concerned, in this study, the influences of the non-linearity of wave loading, the physical properties of backfill materials and the geometry profile of trenches on the excess pore pressures within the soil around pipeline, respectively, were explored, taking into account the in situ conditions of buried pipeline in the shallow ocean zones. Based on the parametric study, it is concluded that the shear modulus and permeability of backfill soils significantly affect the wave-induced excess pore pressures around trenched pipeline, and that the effect of wave non-linearity becomes more pronounced and comparable with that of trench depth, especially at high wave steepness in shallow water.
\end{abstract}

(c) 2005 Elsevier Ltd. All rights reserved.

Keywords: Submarine pipeline; Non-linear wave; Pore pressure; Finite element model

\footnotetext{
* Corresponding author. Fax: +86106256 1284.

E-mail address: fpgao@imech.ac.cn (F.-P. Gao).
}

0029-8018/\$ - see front matter (C) 2005 Elsevier Ltd. All rights reserved. 


\section{Introduction}

Wave-induced forces upon submarine pipelines have attracted extensive attention from coastal engineers, for the ever-increasing engineering activities in offshore and coastal regions. To reduce wave-induced forces and thereby to enhance their stability, offshore pipelines are always trenched into seabed. The trenches are generally backfilled either by in situ sediment or by pouring selected backfill material over the pipeline from bottomdump barge. When water waves propagate over a porous seabed, cyclic excess pore pressures can be generated within seabed with accompanying decrease in effective stresses, which have been recognized as dominant factors for the instability of a buried pipeline. Thus, a proper evaluation of the wave-induced excess pore pressures around pipeline is important for offshore geotechnical engineers involved with the design of foundations for offshore pipeline.

There have been various analytical investigations of the problem of seabed response to wave loading, based on different assumptions of the rigidity of soil skeleton and compressibility of pore fluid, including Madsen (1978), Yamamoto et al. (1978), Mei and Foda (1981), and Jeng (1997). Besides the development of analytical solutions, numerical simulations have been widely applied to examine such a problem in recent years, such as the finite difference method (Zen and Yamazaki, 1990), the finite element method (Thomas, 1989; Gatmiri, 1990; Jeng and Lin, 1996). Most previous investigations of the wave-seabed interaction have been reviewed by Jeng (2003). However, all aforementioned investigations have only examined the soil response under the action of twodimensional progressive waves in the absence of a marine structure.

The importance of wave-soil-pipeline interaction phenomenon has ever been addressed in the literature (Clukey et al., 1989). To date, this problem has not yet been fully understood because of the complicated behavior of soils and the special geometry of pipeline. Design of marine pipelines regarding their stability is a rather complicated problem. Based on the potential theory, the hydrodynamic uplift forces on the buried pipelines have been studied (MacPherson, 1978; Spierenburg, 1986; MacDougal et al., 1988). However, the potential theory is somewhat far from the realistic conditions of soil as a two-phase medium. Based on Biot's consolidation theory (Biot, 1941), the waveinduced pore pressure around a buried pipeline has been studied through a boundary integral equation method (Cheng and Liu, 1986) and a finite element method (Magda, 1997). Among these, Cheng and Liu (1986) considered a buried pipe in a region that is surrounded by two impermeable walls. Magda (1997) considered a similar case with a wider range of the degree of saturation. Jeng (2001) proposed a 2D FEM model to investigate the wave-induced pore pressure, effective stresses within soil around buried pipeline. However, most previous investigations of the wave-seabed-pipe interaction problem have been concerned only with a single sediment layer and linear wave loading. In engineering practice, pipelines are always trenched and covered with coarser materials, especially in shallow water. Moreover, the actual waves in shallow water zone are always characterized as non-linear. However, the non-linear wave-induced responses of backfill soils around trenched pipeline have not been revealed yet.

This paper is aimed at developing a finite element model for investigating the effects of backfill soil properties on non-linear wave-induced excess pore pressures upon a trenched 
pipeline, which will be verified with existing experimental results. The effects of nonlinear component of wave loading are examined firstly. Moreover, the influences of the physical properties of backfill materials and the geometry profile of trenches are thereafter numerically evaluated, respectively, by varying shear modulus, permeability coefficient of backfill soil, slope angle and depth of trenches, etc.

\section{Governing equations and boundary conditions}

In this study, a two-dimensional problem is considered. A pipeline (with outer diameter of $D$ ) is fully buried within a trench in a porous seabed of finite thickness $h$ laid upon an impermeable rigid bottom, as depicted in Fig. 1. The geometry profile of the trench is characterized by trench depth $S$, bottom width $B$ and slope angle $\phi$. The wave is assumed to propagate in the positive $x$-direction, while the $z$-direction is upward from the interface between porous seabed and impermeable rigid bottom.

\subsection{Governing equations}

\subsubsection{Governing equations for porous flows in a seabed}

In this study, the poroelastic theory developed by Biot (1941) is accepted as the governing equation for flow of a compressible pore fluid in a compressible porous medium. The basic assumption is that soil has linear, reversible, isotropic non-retarded

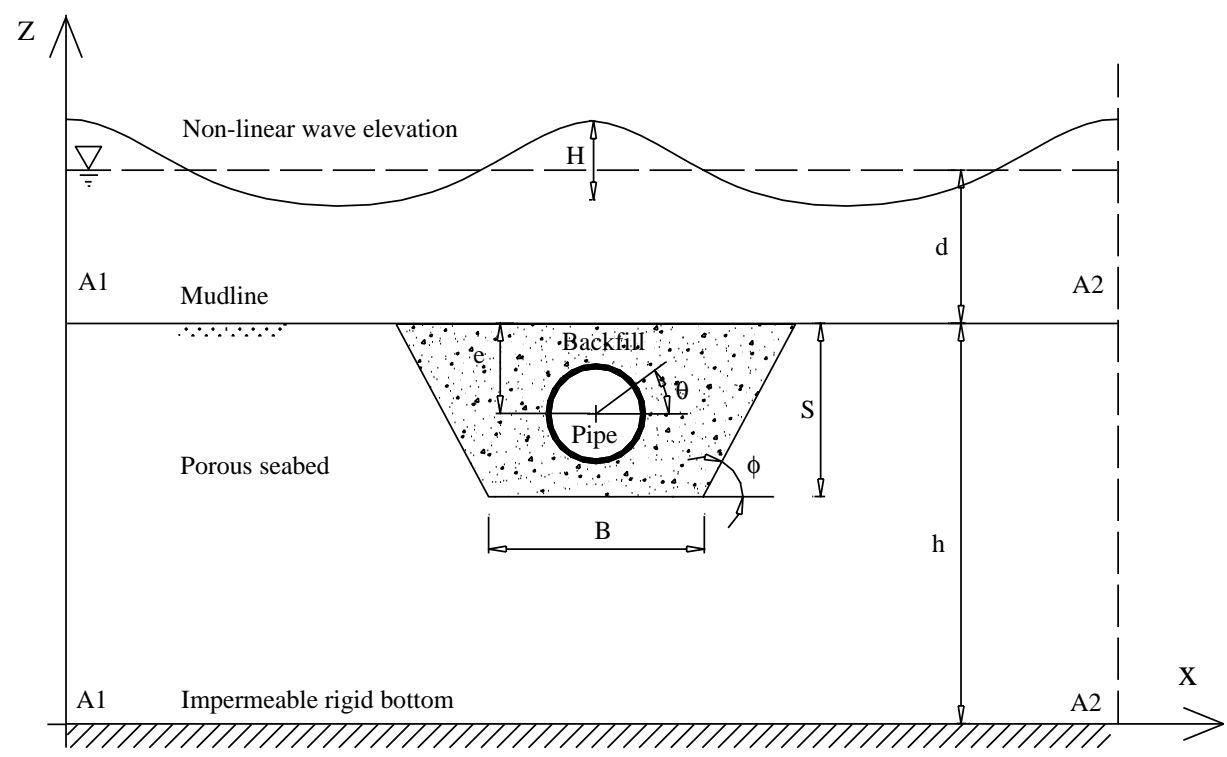

Fig. 1. Definition of wave-seabed-pipeline interaction problem. 
mechanical properties. The movement of pore fluid is assumed to obey Darcy's law. Due to that small oscillatory deformation relative to the hydrostatics equilibrium state is considered; such an idealized assumption may be reasonable. For a two-dimensional case, Biot's equation can be written as

$$
\frac{k}{\gamma_{\mathrm{w}}} \nabla^{2} p-\frac{n}{K^{\prime}} \frac{\partial p}{\partial t}=\frac{\partial}{\partial t}\left(\nabla \vec{u}_{\mathrm{s}}\right),
$$

where $p$ is the excess pore pressure, $k$ is the coefficient of soil permeability, $\gamma_{\mathrm{w}}$ is the unit weight of pore water, $n$ is the porosity of soil, $t$ is the time, $\vec{u}_{s} \equiv\left(u_{s}, w_{s}\right)$ is the vector of soil displacements, $K^{\prime}$ is the apparent bulk modulus of pore water

$$
\frac{1}{K^{\prime}}=\frac{1}{K_{\mathrm{w}}}+\frac{1-S_{\mathrm{r}}}{P_{\mathrm{w} 0}},
$$

in which $K_{\mathrm{w}}$ is the true modulus of elasticity of water (taken as $2 \times 10^{9} \mathrm{~Pa}$ ), $P_{\mathrm{w} 0}$ is the absolute water pressure and $S_{\mathrm{r}}$ is the saturation degree of soil.

Neglecting the effects of body forces and inertia terms, the equations governing the overall equilibrium of a porous medium can be expressed in terms of pore pressure and soil displacements as

$$
\begin{aligned}
& G \nabla^{2} u_{\mathrm{s}}+\frac{G}{1-2 \mu} \frac{\partial}{\partial x}\left(\nabla \vec{u}_{\mathrm{s}}\right)=-\frac{\partial p}{\partial x} \\
& G \nabla^{2} w_{\mathrm{s}}+\frac{G}{1-2 \mu} \frac{\partial}{\partial x}\left(\nabla \vec{u}_{\mathrm{s}}\right)=-\frac{\partial p}{\partial z}
\end{aligned}
$$

in the $x$ and $z$ directions, respectively.

Under conditions of plane strain, the effective stresses are related to the strains by Hook's law as

$$
\begin{aligned}
& \sigma_{x}^{\prime}=2 G(z)\left[\frac{\partial u_{\mathrm{s}}}{\partial x}+\frac{\mu}{1-2 \mu}\left(\nabla \vec{u}_{\mathrm{s}}\right)\right], \\
& \sigma_{z}^{\prime}=2 G(z)\left[\frac{\partial w_{\mathrm{s}}}{\partial z}+\frac{\mu}{1-2 \mu}\left(\nabla \vec{u}_{\mathrm{s}}\right)\right], \\
& \tau_{x z}=G(z)\left[\frac{\partial u_{\mathrm{s}}}{\partial z}+\frac{\partial w_{\mathrm{s}}}{\partial x}\right]=\tau_{z x},
\end{aligned}
$$

where $G$ is the shear modulus of soil, which is related to Young's modulus $(E)$ and Poisson's ratio $(\mu)$ as $E / 2(1+\mu)$. It is noted that a positive sign is taken for a compressive normal stress in this study.

\subsubsection{Governing equations for a trenched pipeline}

In this study, we consider the trenched pipeline is an elastic material. With the absence of the body forces, based on the minimum potential energy theorem, the total potential 
energy of the buried pipe can be expressed as

$$
\Pi=\frac{1}{2} \sigma_{\mathrm{p}, i j} \varepsilon_{\mathrm{p}, i j} \mathrm{~d} V-\int_{A} f_{i} u_{\mathrm{p}, i} \mathrm{~d} A,
$$

in which $\sigma_{\mathrm{p}}$ and $\varepsilon_{\mathrm{p}}$ are internal stress and strain within pipeline wall, respectively, $f$ is the wave-induced forces acting the buried pipeline, and $u_{\mathrm{p}}$ is the pipeline deformation induced by external forces. Applying the variational method to (8), we have

$$
\delta \Pi=\frac{1}{2} \sigma_{\mathrm{p}, i j} \delta \varepsilon_{\mathrm{p}, i j} \mathrm{~d} V-\int_{A} f_{i} \delta u_{\mathrm{p}, i} \mathrm{~d} A .
$$

According to the minimum energy theorem (i.e. $\delta \Pi=0)$, (9) can be written as

$$
\frac{1}{2} \int_{V} \sigma_{\mathrm{p}, i j} \delta \varepsilon_{\mathrm{p}, i j} \mathrm{~d} V=\int_{A} f_{i} \delta u_{\mathrm{p}, i} \mathrm{~d} A .
$$

Since we assume there is no relative movement between pipe and soil particles at the pipeline outer surface, the wave-induced soil deformation is also included in terms of pore pressure by (1), (3) and (4). Also, the shear stresses and normal stresses are linked with soil displacements by (5)-(7). Thus, while we solve the soil response around the pipeline with internal stresses, the shear stress, normal stresses and soil displacements are transferred (they are directly affected by pore pressure) into pore pressures as the external loading in the evaluation of the internal stresses within the pipeline.

\subsection{Boundary conditions}

For a porous seabed of finite thickness, as shown in Fig. 1, the evaluation of the waveinduced seabed response requires the solution of (1), (3), (4) and (10), together with the appropriate boundary conditions.

\subsubsection{Bottom boundary conditions (BBC)}

For the soil resting on an impermeable rigid base, zero displacements and no vertical flow occur at the interface between the soil and the impermeable rigid bottom, i.e.

$$
u_{\mathrm{s}}=w_{\mathrm{s}}=\frac{\partial p}{\partial z}=0, \quad \text { at } z=0 .
$$

\subsubsection{Seabed surface conditions (SSC)}

The wave-induced frictional stress at the surface of seabed is small and negligible, compared to the high-dynamic pore pressures. Thus the vertical effective normal stress and shear stress vanish at the surface of seabed

$$
\sigma_{z}^{\prime}=\tau_{x z}=0, \text { at } z=d .
$$

The pore pressure at the surface of the seabed is equal to the wave pressure induced by the progressive wave. It has been agreed that the wave profile has steep crest and flat 
trough in shallow water results from its strong non-linearity. Laitone (1962) has investigated the range of validity of Stokes non-linear wave theory on a theoretical basis, and suggested that it is most suitable for shallow water zones where wavelengths are less than about eight times the water depth. In this study, second-order approximation of wave pressure based on Stokes wave theory in two-dimensional problems is employed, i.e.

$$
p=P_{\mathrm{a}} \cos (2 \pi x / L-\omega t)+\frac{\pi \rho g H^{2}}{2 L}\left[A_{21}+A_{22} \cos 2(2 \pi x / L-\omega t)\right], \quad \text { at } z=h,
$$

where $H$ is the wave height, $d$ is the water depth, $L$ is the wave length, $\omega$ is the wave frequency ( $=2 \pi / T, T$ is the wave period), $P_{\mathrm{a}}$ is the amplitude of wave pressure based on Airy linear wave theory, i.e.

$$
\begin{aligned}
& P_{\mathrm{a}}=\frac{\rho g H}{2 \cosh (2 \pi d / L)}, \\
& A_{21}=-\omega_{0} \beta_{1}-\frac{\omega_{0}^{2}}{4 \sinh ^{2} 2 \pi d / L}, \quad A_{22}=4 \omega_{0} \beta_{2}-\frac{\omega_{0}^{2}}{4 \sinh ^{2} 2 \pi d / L}, \\
& \omega_{0}=\sqrt{\tanh 2 \pi d / L}, \quad \beta_{1}=\frac{1}{8}\left(-\omega_{0}^{-3}+\omega_{0}\right), \quad \beta_{2}=\frac{3\left(\omega_{0}^{-7}+\omega_{0}\right)}{16 \cosh (4 \pi d / L)}
\end{aligned}
$$

Eq. (13) can also be written in the following form

$$
\begin{aligned}
p= & p_{0}+p_{1} \operatorname{Re}\left\{(\cos 2 \pi x / L+\mathrm{i} \sin 2 \pi x / L) \mathrm{e}^{-\mathrm{i} \omega t}\right\}+p_{2} \operatorname{Re}\{(\cos 4 \pi x / L \\
& \left.+\mathrm{i} \sin 4 \pi x / L) \mathrm{e}^{-\mathrm{i} 2 \omega t}\right\}
\end{aligned}
$$

in which $p_{0}=\left(\pi \rho g H^{2} / 2 L\right) A_{21}, p_{1}=P_{\mathrm{a}}, p_{2}=\left(\pi \rho g H^{2} / 2 L\right) A_{22}$, 'Re' represents the real part of the complex number in bracket.

\subsubsection{Boundary conditions of pipeline $(P B C)$}

It is noted that most of previous studies of wave-seabed-pipe interaction have assumed the pipeline to be rigid (Cheng and Liu, 1986; Magda, 1997). In this study, pipeline is assumed as an elastic medium. Thus, the boundary conditions along the pipeline inner surface are kept free. Besides the assumption that there is no relative movement between pipe and soil particles at the pipeline outer surface, it is reasonable to assume there is no flow through the pipeline wall. Therefore, the pressure gradient should vanish at the surface of the pipe, i.e.

$$
\frac{\partial p}{\partial n}=0, \quad \text { at } r=\sqrt{\left(x-x_{0}\right)+\left(z-z_{0}\right)}=D / 2 .
$$

\subsubsection{Backfill-seabed interface conditions (BSC)}

The wave-induced excess pore pressures and soil displacements at the interface between the backfill soil and the seabed are assumed identical (see Fig. 1), i.e. no relative displacement occurs and the pore pressure is continuous at the interface. 


\subsubsection{Lateral boundary conditions ( $L B C)$}

It can be seen from Eq. (15) that second-order non-linear wave loading upon seabed is periodical. The wave-seabed interaction problem can be solved by employing the principle of repeatability (Zienkiewicz and Scott, 1972). As shown in Fig. 1, the waveinduced excess pore pressure and soil displacements at sections A1-A1 and A2-A2 should be identical, i.e.

$$
\begin{aligned}
& p\left(x \text { at ' } A 1-A 1^{\prime}, z, t\right)=p(x \text { at ' } A 2-A 2 \text { ', } z, t), \\
& u_{\mathrm{s}}\left(x \text { at ' } A 1-A 1^{\prime}, z, t\right)=u_{\mathrm{s}}(x \text { at ' } A 2-A 2 \text { ', } z, t), \\
& w_{\mathrm{s}}\left(x \text { at ' } A 1-A 1^{\prime}, z, t\right)=w_{\mathrm{s}}(x \text { at ' } A 2-A 2 \text { ', } z, t) .
\end{aligned}
$$

This concept is particularly convenient for periodical loading such as the present problem.

\section{Finite element model}

\subsection{Finite element formulations for porous seabed}

The second-order non-linear wave-induced oscillatory soil response is periodically fluctuating in the temporal domain. Similar to expression form of wave pressure at the surface of seabed in Eq. (15), the wave-induced excess pore pressure, soil displacements and effective stresses within seabed can be expressed as

$$
\begin{aligned}
Q_{j}(x, y, t)= & Q_{j 0 \mathrm{r}}(x, y, t)+\left[Q_{j 1 \mathrm{r}}(x, y, t)+\mathrm{i} Q_{j 1 \mathrm{c}}(x, y, t)\right] \mathrm{e}^{-\mathrm{i} \omega t}+\left[Q_{j 2 \mathrm{r}}(x, y, t)\right. \\
& \left.+\mathrm{i} Q_{j 2 \mathrm{c}}(x, y, t)\right] \mathrm{e}^{-\mathrm{i} 2 \omega t}
\end{aligned}
$$

where $Q_{j}(j=1, \ldots, 6)$ represents $p, u_{\mathrm{s}}, w_{\mathrm{s}}, \sigma_{x}^{\prime}, \sigma_{y}^{\prime}$ and $\tau_{x y}$, respectively. Subscripts 'r' and 'c' represent the real and imaginary parts of the soil response. Substituting Eq. (18) into Eqs. (1), (3) and (4), then directly applying the Galerkin method (Zienkiewicz and Taylor, 1989) to these equations, the finite element analytical formulations can be expressed in a matrix form as

$$
\begin{aligned}
& \int_{S} N_{i}\left[Q_{\mathrm{e}}\right] \mathrm{d} S=\int_{V} B_{1}^{\mathrm{T}} D_{1} B_{1} \mathrm{~d} V[P]+\int_{V} B_{2}^{\mathrm{T}} D_{2} B_{2} \mathrm{~d} V[P]+\int_{V} B_{3}^{\mathrm{T}} D_{3} B_{3} \mathrm{~d} V\left[U_{\mathrm{s}}\right], \\
& \int_{S} N_{i}\left[F_{\mathrm{e}}\right] \mathrm{d} S=\int_{V} B_{4}^{\mathrm{T}} D_{4} B_{4} \mathrm{~d} V\left[U_{\mathrm{s}}\right]+\int_{V} B_{5}^{\mathrm{T}} B_{1} \mathrm{~d} V[P],
\end{aligned}
$$

where

$$
\left[Q_{\mathrm{e}}\right]=\left[\begin{array}{ccccc}
\left(q_{n \mathrm{r}}\right)_{1} & 0 & \cdots & \left(q_{n \mathrm{r}}\right)_{n_{\mathrm{e}}} & 0 \\
0 & \left(q_{n \mathrm{c}}\right)_{1} & \cdots & 0 & \left(q_{n \mathrm{c}}\right)_{n_{\mathrm{e}}}
\end{array}\right],
$$




$$
\begin{aligned}
& {\left[F_{\mathrm{e}}\right]=\left[\begin{array}{ccccccccc}
\left(f_{x \mathrm{r}}\right)_{1} & 0 & \left(f_{\mathrm{yr}}\right)_{1} & 0 & \cdots & \left(f_{x \mathrm{r}}\right)_{n_{\mathrm{e}}} & 0 & \left(f_{y \mathrm{r}}\right)_{n_{\mathrm{e}}} & 0 \\
0 & \left(f_{x \mathrm{c}}\right)_{1} & 0 & \left(f_{y \mathrm{c}}\right)_{1} & \cdots & 0 & \left(f_{x \mathrm{c}}\right)_{n_{\mathrm{e}}} & 0 & \left(f_{y \mathrm{c}}\right)_{n_{\mathrm{e}}}
\end{array}\right],} \\
& \left(\left\{\begin{array}{l}
q_{n \mathrm{r}} \\
q_{n \mathrm{c}}
\end{array}\right\}\right)_{i}=\left(\frac{k}{\gamma_{\mathrm{w}}}\left\{\begin{array}{c}
\frac{\partial p_{\mathrm{r}}}{\partial x} \\
\frac{\partial p_{\mathrm{c}}}{\partial x}
\end{array}\right\} n_{x}+\frac{k}{\gamma_{\mathrm{w}}}\left\{\begin{array}{c}
\frac{\partial p_{\mathrm{r}}}{\partial z} \\
\frac{\partial p_{\mathrm{c}}}{\partial z}
\end{array}\right\} n_{z}\right)_{i} \\
& \left(\left\{\begin{array}{c}
f_{x \mathrm{r}} \\
f_{x \mathrm{c}} \\
f_{z \mathrm{r}} \\
f_{z \mathrm{c}}
\end{array}\right\}\right)_{i}=\left(\left\{\begin{array}{c}
\sigma_{x \mathrm{r}}^{\prime} \\
\sigma_{x \mathrm{c}}^{\prime} \\
\tau_{x z \mathrm{r}} \\
\tau_{x z \mathrm{c}}
\end{array}\right\} n_{x}+\left\{\begin{array}{c}
\tau_{x z \mathrm{r}} \\
\tau_{x z \mathrm{c}} \\
\sigma_{x \mathrm{r}}^{\prime} \\
\sigma_{x \mathrm{c}}^{\prime}
\end{array}\right\} n_{z}\right)_{i} \\
& {[P]=\left[\begin{array}{ccccc}
\left(p_{\mathrm{r}}\right)_{1} & 0 & \cdots & \left(p_{\mathrm{r}}\right)_{n_{\mathrm{e}}} & 0 \\
0 & \left(p_{\mathrm{c}}\right)_{1} & \cdots & 0 & \left(p_{\mathrm{c}}\right)_{n_{\mathrm{e}}}
\end{array}\right]} \\
& {\left[U_{\mathrm{s}}\right]=\left[\begin{array}{ccccccccc}
\left(u_{\mathrm{sr}}\right)_{1} & 0 & \left(w_{\mathrm{sr}}\right)_{1} & 0 & \cdots & \left(u_{\mathrm{sr}}\right)_{n_{\mathrm{e}}} & 0 & \left(w_{\mathrm{sr}}\right)_{n_{\mathrm{e}}} & 0 \\
0 & \left(u_{\mathrm{sc}}\right)_{1} & 0 & \left(w_{\mathrm{sc}}\right)_{1} & \cdots & 0 & \left(u_{\mathrm{sc}}\right)_{n_{\mathrm{e}}} & 0 & \left(w_{\mathrm{sc}}\right)_{n_{\mathrm{e}}}
\end{array}\right]}
\end{aligned}
$$

where $n_{\mathrm{e}}$ is the number of nodes per element, $N_{i}$ is the shape function of the $i$ th node, $p_{\mathrm{r}}$, and $w_{\mathrm{s}}$ are in form of Eq. (18), and coefficient matrices $B_{i}$ and $D_{i}$ are given in Appendix A.

\subsection{Finite element formulations for a trenched pipeline}

Similarly, the second-order non-linear wave-induced internal stresses $\left(\sigma_{\mathrm{p} x}, \sigma_{\mathrm{p} z}, \tau_{\mathrm{p} x z}\right)$ and deformations of the pipe $\left(u_{\mathrm{p}}, w_{\mathrm{p}}\right)$ and the external forces $\left(f_{\mathrm{p}}\right)$ can be expressed as

$$
\begin{aligned}
G_{j}(x, y, t)= & G_{j 0 \mathrm{r}}(x, y, t)+\left[G_{j 1 \mathrm{r}}(x, y, t)+\mathrm{i} G_{j 1 \mathrm{c}}(x, y, t)\right] \mathrm{e}^{-\mathrm{i} \omega t}+\left[G_{j 2 \mathrm{r}}(x, y, t)\right. \\
& \left.+\mathrm{i} G_{j 2 \mathrm{c}}(x, y, t)\right] \mathrm{e}^{-\mathrm{i} 2 \omega t}
\end{aligned}
$$

where $G_{j}(j=1, \ldots, 6)$ represents $f_{\mathrm{p}}, u_{\mathrm{p}}, w_{\mathrm{p}}, \sigma_{\mathrm{p} x}, \sigma_{\mathrm{p} z}$ and $\tau_{\mathrm{p} x z}$, respectively. Introducing Eq. (27) into Eq. (10), we have

$$
\int_{S} N_{i}\left[F_{\mathrm{p}}\right] t_{\mathrm{p}} \mathrm{d} S=\int_{A} B_{5}^{\mathrm{T}} D_{5} B_{5}\left[U_{\mathrm{p}}\right] t_{\mathrm{p}} \mathrm{d} A,
$$

where $U_{\mathrm{p}}$ is the nodal displacement matrix of pipe, $t_{\mathrm{p}}$ is the thickness of the pipeline and $f_{\mathrm{p}}$ is the force matrix acting on the pipe, and the matrices $D_{1}-D_{5}$ are given in Appendix A.

To improve the accuracy of the solution for wave-pipeline-seabed interaction, two different mesh systems are used in the present model. As seen in Fig. 2, an eight-node 


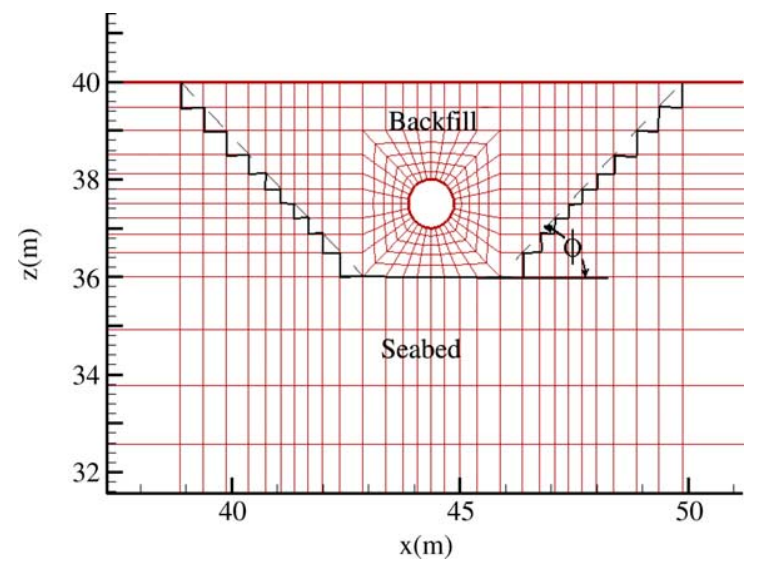

Fig. 2. Finite-element mesh in the vicinity of the pipeline.

iso-parametric element is used in the region near the pipeline. Outside this region, an eight-nodal rectangular element is used.

\section{Verification of the numerical model}

To verify the aforementioned non-linear wave-soil-pipe interaction numerical model, a comparison of the numerical results with the experimental results by Sudhan et al. (2002) and Turcotte et al. (1984) is carried out, as shown in Fig. 3. The tests by Sudhan et al. (2002) were conducted in a $30 \mathrm{~m}$ long, $2 \mathrm{~m}$ wide and $1.7 \mathrm{~m}$ deep wave flume.

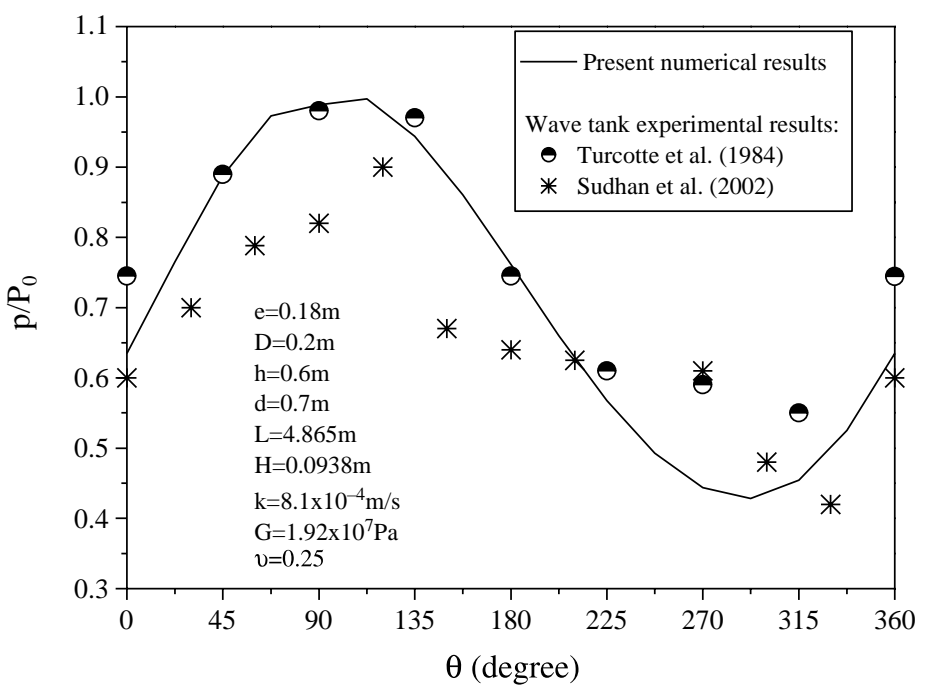

Fig. 3. Verification of numerical model with test results. 
The aluminum pipe $200 \mathrm{~mm}$ in diameter, $1.96 \mathrm{~m}$ in length with $10 \mathrm{~mm}$ thick walls was buried within sand pit $2.0 \mathrm{~m} \times 2.0 \mathrm{~m} \times 0.6 \mathrm{~m}$ in size, where the uniform and fairy homogeneous soil bed was formed by using a standard raining technique. The main parameters of sand, pipe and wave loading are given in Fig. 3. At the center of the pipe, 12 miniature pressure transducers were placed on the outer periphery of the pipe for measuring the wave-induced pore pressure. It is noted that the test results reported by Turcotte et al. (1984) are obtained for almost similar conditions of burial depth and wave conditions and soil properties with those of Sudhan et al. (2002). The wave-induced pore pressure distributions along pipe surface under the phase of wave crest are plotted in Fig. 3. It is indicated from the figure that the numerical results agree overall with the experimental data. The numerical model is capable of catching the trends for variations of wave-induced pore pressure, $p / P_{0}$, with $\theta$ recorded in the experiments.

\section{Parametric study and discussion}

In this study, the above FEM model is used to investigate the transient response of soil around trenched pipeline under non-linear wave loading. The input data for the investigations are listed in Table 1.

Table 1

Input data for parametric study

Wave characteristics

Wave period $(T)$

Water depth $(d)$

Wave length $(L)$

Wave height $(H)$

Pipeline characteristics

Poisson ratio $\left(\nu_{\mathrm{p}}\right)$

Young's modulus $\left(E_{\mathrm{p}}\right)$

Pipe outer diameter $(D)$

Pipe thickness $\left(t_{\mathrm{p}}\right)$

Density of pipe $\left(\rho_{\mathrm{p}}\right)$

Seabed characteristics

Seabed thickness $(h)$

Poisson ratio $(\nu)$

Porosity (n)

Shear modulus $(G)$

Permeability $(k)$

Degree of saturation $\left(S_{\mathrm{r}}\right)$

Backfill soil characteristics

Shear modulus $(G)$

Permeability $(k)$

Trench bottom width $(B)$

Trench slope angle $(\phi)$

Trench depth $(S)$

Buried depth of pipe $(e)$

(Values of $\nu, n, S_{\mathrm{r}}$ are same as those of seabed)

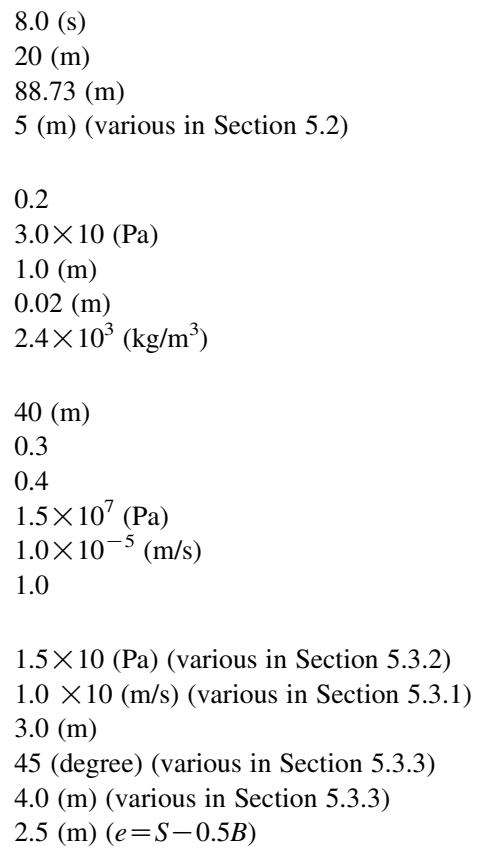




\subsection{Transient pore pressure response of soil along pipeline at various phases} of non-linear progressive waves

For the study of transient response of soil around pipeline at various phases of progressive waves, the calculation zone between sections A1-A1 and A2-A2 in Fig. 1 is chosen as two times of wavelengths $(2 L)$, and the pipeline is located at $x=(1.5-t / T) L$ for various phases, e.g. wave tough (when $t=0$ ), wave crest (when $t=0.5 T$ ) as shown in Fig. 4.

Fig. 5 gives the non-linear wave-induced pore pressure along pipeline buried in a homogenous fine sand seabed $\left(k=1.0 \times 10^{-5} \mathrm{~m} / \mathrm{s}\right)$ at various phases of non-linear progressive wave. As indicated in Figs. 4 and 5, an over-pressure with respect to the initial hydrostatic pressure distribution defined by the still water level generated in the soil due to a passage of a wave crest creates positive excess pore pressure along buried pipeline. On the contrary, an under-pressure induced by a passage of a wave trough creates passive excess pore pressures. For each moment of wave oscillations, the spatial distribution of excess pore pressure along pipeline surface will result in the seepage force upon pipeline, which would have influence upon vertical stability of buried pipeline. Unlike the soil response under linear wave loading, the amplitude of pore pressure under wave crest is bigger than that under wave trough. Therefore, the bottom pressure fluctuations induced by non-linear waves have a direct and continuous influence on changes in pore pressure along pipeline.

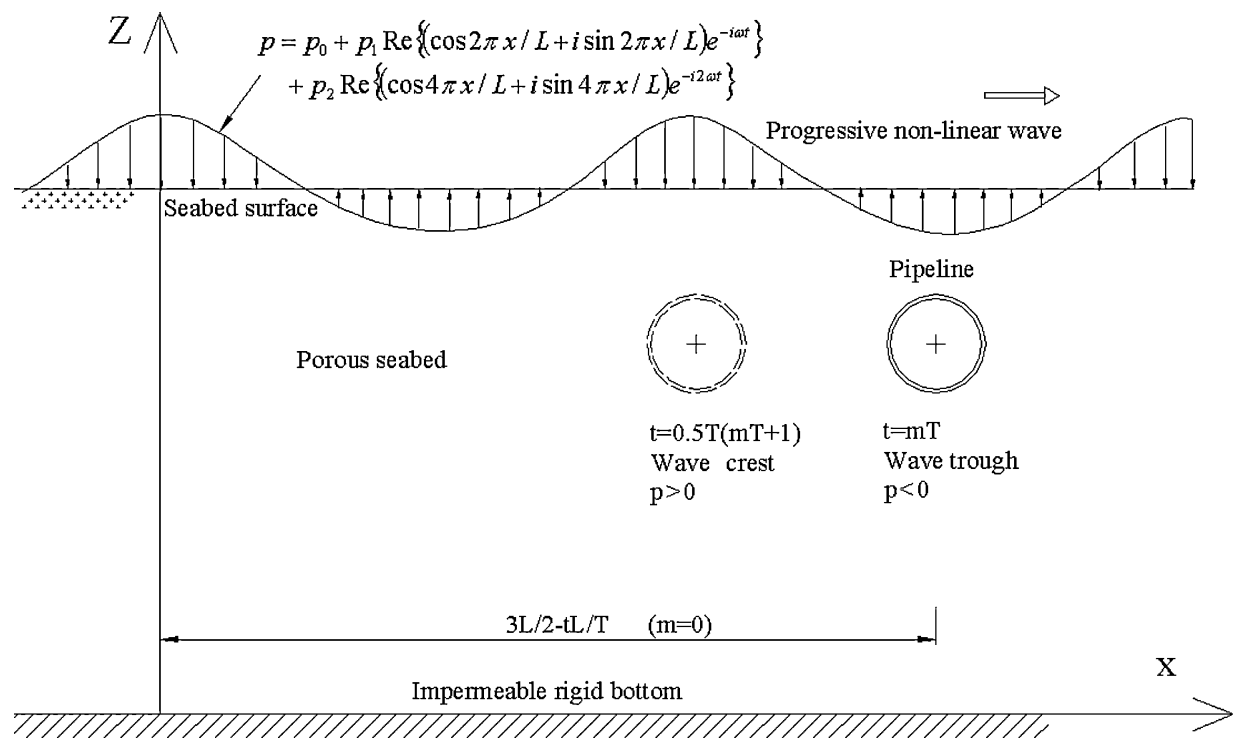

Fig. 4. Sketch of non-linear wave-induced pore pressure under two characteristic phases, i.e. wave crest and wave trough of progressive wave oscillations. 


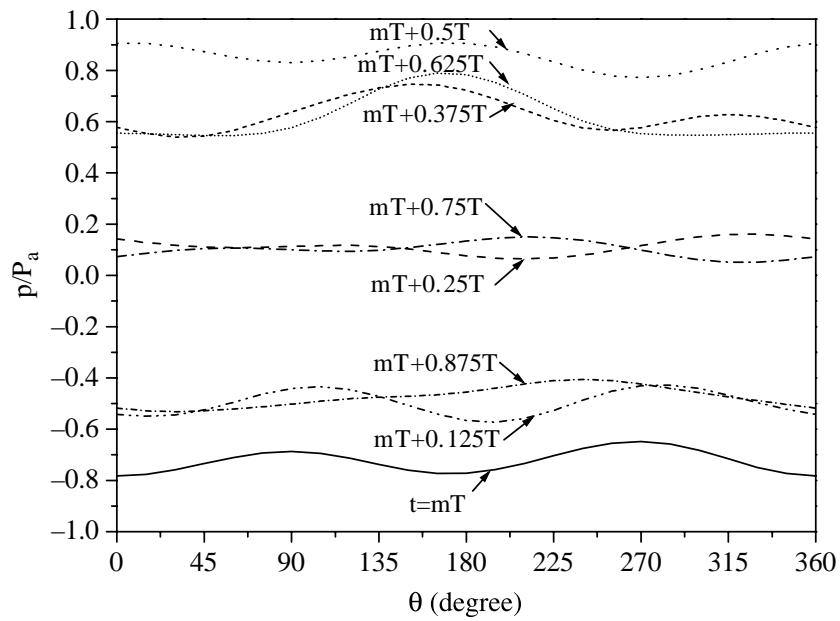

Fig. 5. Wave-induced pore pressure along pipeline buried in homogenous fine sand $\left(k=1.0 \times 10^{-5} \mathrm{~m} / \mathrm{s}\right)$ at various phases of non-linear progressive wave.

\subsection{Effect of non-linear component of wave loading}

For the fixed values of the wavelength, water depth and the properties of seabed and pipeline, the effects of non-linear component of wave loading at wave crest phase are examined by varying wave height, i.e. $H=1$ (wave steepness $H / L=0.011), 3(H / L=$ $0.034)$ and $5 \mathrm{~m}(H / L=0.056)$. Fig. 6 illustrates the distribution of non-linear wave-induced excess pore pressure $\left(p / P_{\mathrm{a}}\right)$ along pipeline trenched in fine sand seabed and backfilled with coarse sand for various wave steepness $(H / L)$. The figure clearly indicates that with the increase of wave steepness, the wave-induced excess pore pressures along pipeline

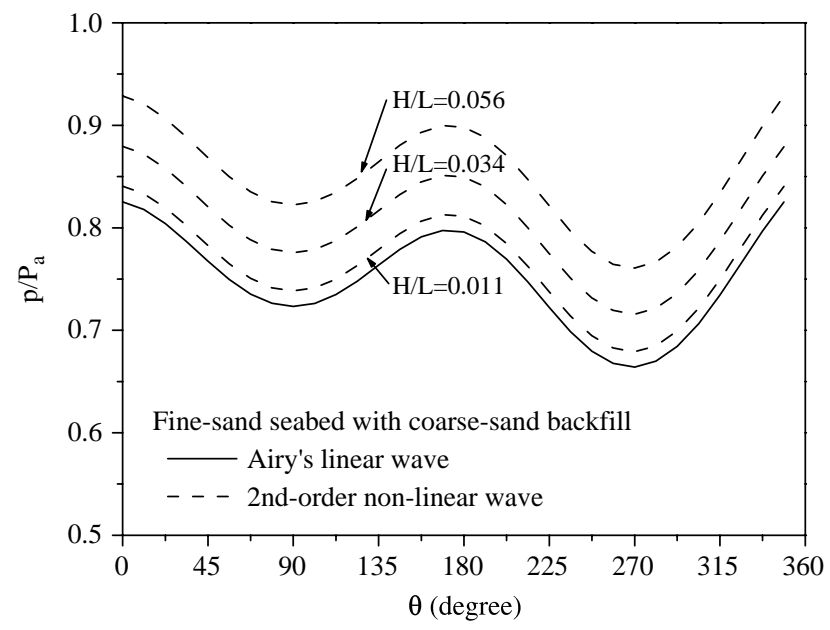

Fig. 6. Effects of wave non-linearity on excess pore pressure in the vicinity of pipeline trenched. 
increase obviously. This implies that the effects of non-linear wave components on the response of soil could not be always ignored without substantial errors, especially for large wave steepness in shallow water. In addition, the effects of wave non-linearity are also investigated by altering the trench depth (see Section 5.3.3).

\subsection{Effect of property of backfill sand}

\subsubsection{Effect of permeability of backfill sand}

In the field, the trenches are often backfilled by selected coarse-grained sand over the pipeline to reduce hydrodynamic loads. In order to investigate the effects of the
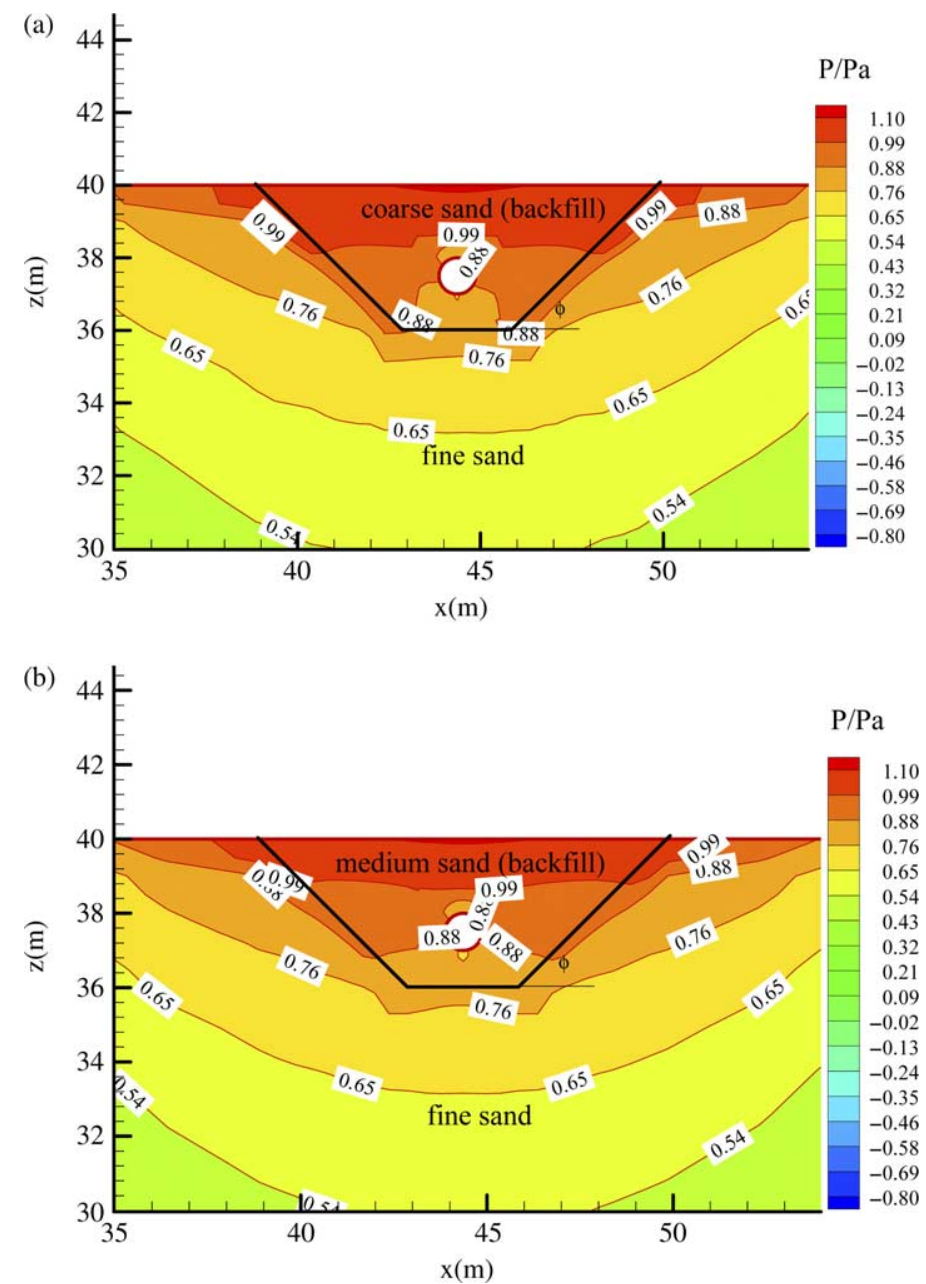

Fig. 7. Contours of non-linear wave-induced excess pore pressure in the vicinity of pipeline backfilled with different sands:(a) $k=1.0 \times 10^{-3} \mathrm{~m} / \mathrm{s}$ (coarse sand), (b) $k=5.0 \times 10^{-4} \mathrm{~m} / \mathrm{s}$ (medium sand). 
permeability of backfill sand upon wave-induced excess pore pressures around pipeline, various permeability coefficients of backfill sand are chosen for the parametric study, i.e. $k=1.0 \times 10^{-3}$ (coarse sand), $5.0 \times 10^{-4}$ (medium sand), $1.0 \times 10^{-5} \mathrm{~m} / \mathrm{s}$ (same as seabed)

The contours of excess pore pressure $\left(p / P_{\mathrm{a}}\right)$ in the vicinity of the pipeline buried in trench and in homogenous seabed under wave crest phase of progressive wave oscillations are illustrated in Figs. 7 and 8, respectively. It is observed that the excess pore pressure around the pipeline is significantly affected by the permeability of backfill. The coarser the backfill sand, the higher the excess pore pressures at the zone near the seabed mudline. Fig. 9 shows the distribution of second-order non-linear wave-induced excess pore pressure $\left(p / P_{\mathrm{a}}\right)$ along pipe surface for the various permeability coefficients of backfill soil. However, the excess pore pressure beneath the pipeline $\left(\theta=270^{\circ}\right)$ gets lower in the coarse backfill sands, meaning that pipeline buried in coarse backfill sands is more vertically stable than in medium/fine sand backfill or in homogenous fine sand seabed.

\subsubsection{Effect of shear modulus of backfill sand}

The in situ sediment is sometimes compacted to desired shear modulus or soil stiffness for pipe bedding and backfill. Normally speaking, the shear modulus of soil $(G)$ increases significantly with the increase of soil density. To investigate the effects of shear modulus of backfill on the soil responses along pipeline, the permeability coefficient is assumed constant when varying the shear modulus of backfill soil.

Fig. 10 illustrates the distribution of non-linear wave-induced excess pore pressure $(p /$ $\left.P_{\mathrm{a}}\right)$ along pipeline for the various shear modulus of backfill soil $(G)$. As shown in the figure, the wave-induced excess pore pressure $p / P_{\mathrm{a}}$ decreases with the increase of $G$. One may suggest that the higher the stiffness, the smaller the strain induced by the same amplitude of wave loading, therefore lower excess pore pressure can be generated.

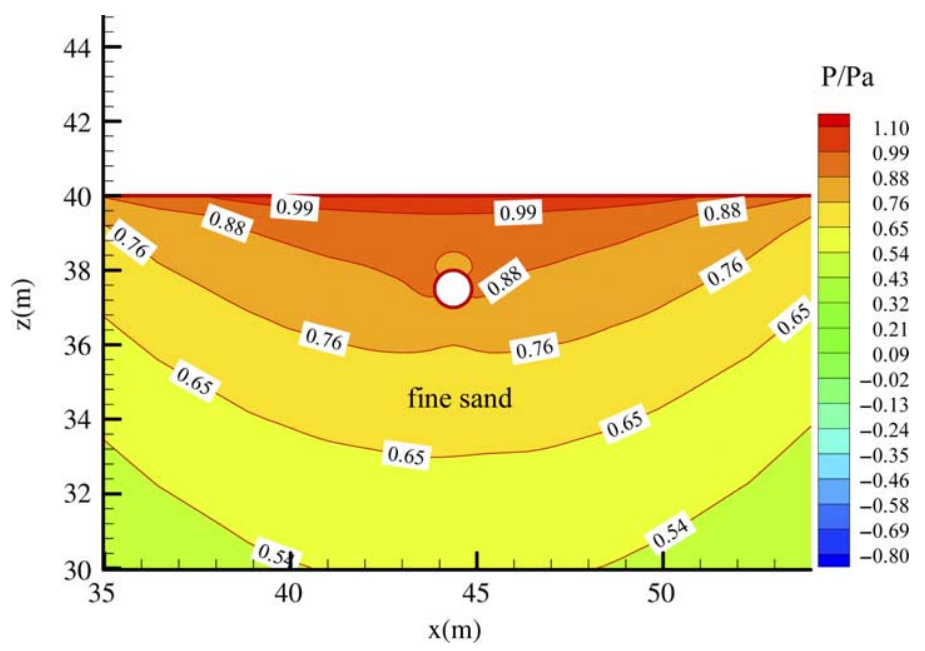

Fig. 8. Contours of non-linear wave-induced excess pore pressure in the vicinity of pipeline buried in homogenous fine sand $\left(k=1.0 \times 10^{-5} \mathrm{~m} / \mathrm{s}\right)$ under wave crest phase of progressive wave oscillations. 


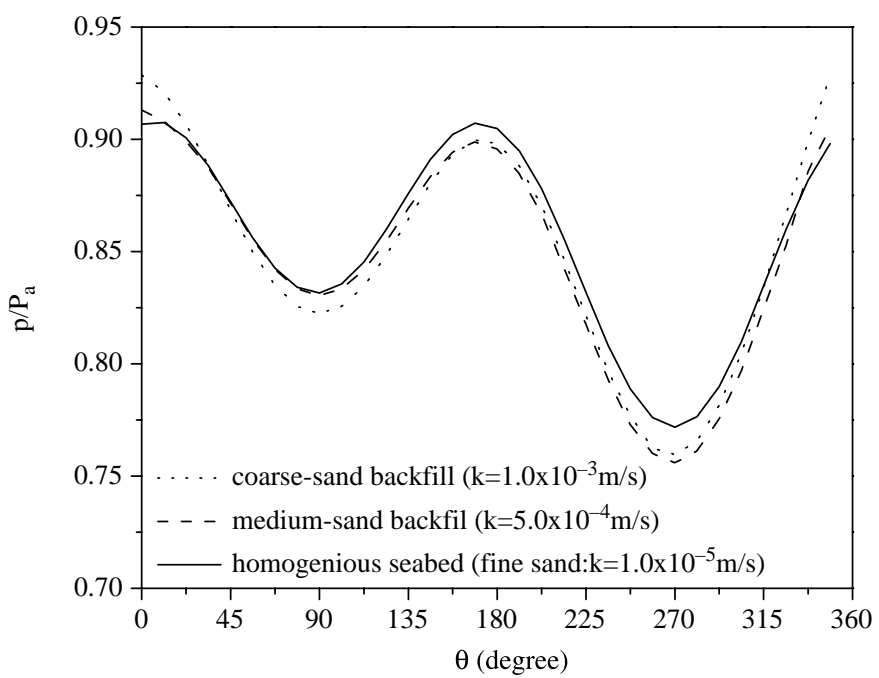

Fig. 9. Distribution of non-linear wave-induced excess pore pressure along pipeline backfilled with sands of various permeability coefficients.

\subsubsection{Effect of the geometry profile of trenches}

As described above, the geometrical profiles of trench can be described by trench depth $(S)$, bottom width $(B)$ and slope angle $(\alpha)$. With the fixed values of $S$ and $B$ as listed in Table 1 , the effects of slope angle $\alpha$ on the wave-induced excess pore pressures around pipeline are investigated by varying the value of $\alpha$, with coarse sand $\left(k=1.0 \times 10^{-3} \mathrm{~m} / \mathrm{s}\right)$ as backfill materials. Fig. 11 shows the distribution of $p / P_{\mathrm{a}}$ along a pipeline surface for various slope angles. As shown in the figure, wave-induced excess pore pressures $\left(p / P_{\mathrm{a}}\right)$ at

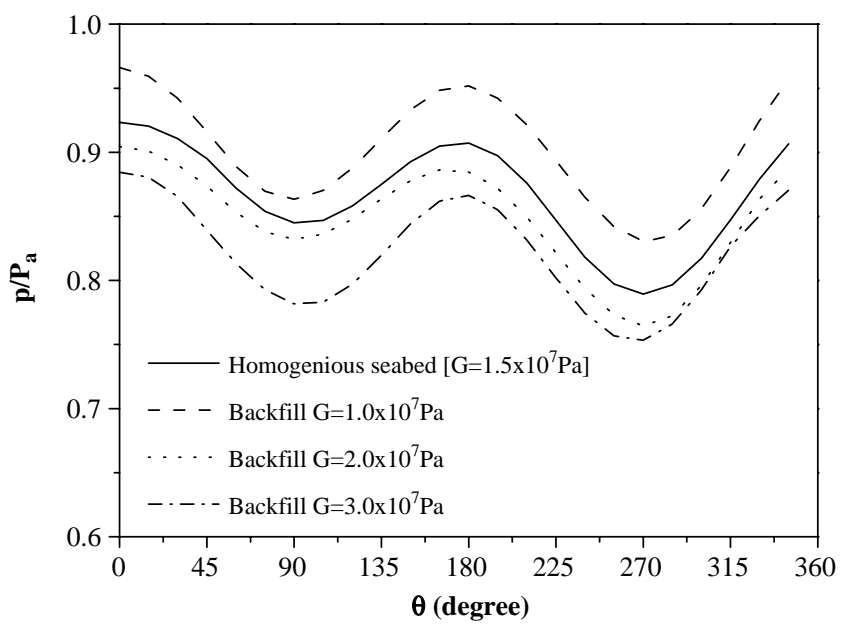

Fig. 10. Distribution of non-linear wave-induced excess pore pressure along pipeline backfilled with sands of various shear modulus. 


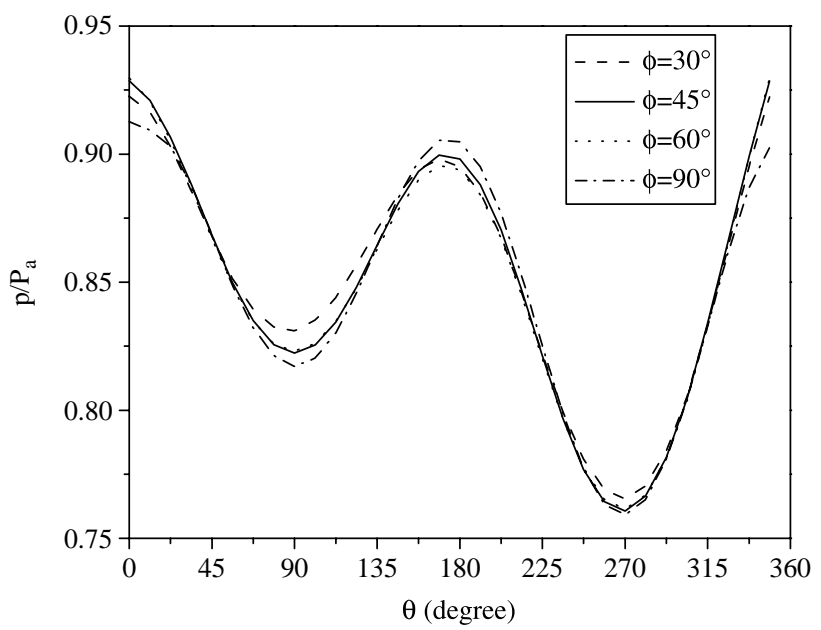

Fig. 11. Distribution of non-linear wave-induced excess pore pressure along trenched pipeline for various slope angles.

top and bottom of pipeline $\left(\theta=90,270^{\circ}\right.$, respectively) increase with the decrease of slope angle from 90 to $30^{\circ}$.

Besides the effects of slop angle, it is also interesting to investigate the effects of another important factor in the design of trench for pipeline burial, i.e. the trench depth $(S)$. In the fields, a backfill cover with the burial depth up to $4 \mathrm{~m}$ in silt and coarse sediments has been used in shallow water zones. In this numerical mesh design, burial depth of pipeline $(e)$ is related to trench depth $(S)$ and trench bottom width $(B)$ by $e=S-$ $0.5 B$. To gain a basic understanding of the effect of $S$ on the distribution of $p / P_{\mathrm{a}}$ around pipeline surface, $S=3.0,4.0$ and $5.0 \mathrm{~m}$ is taken with the fixed values of $D=1.0 \mathrm{~m}$,

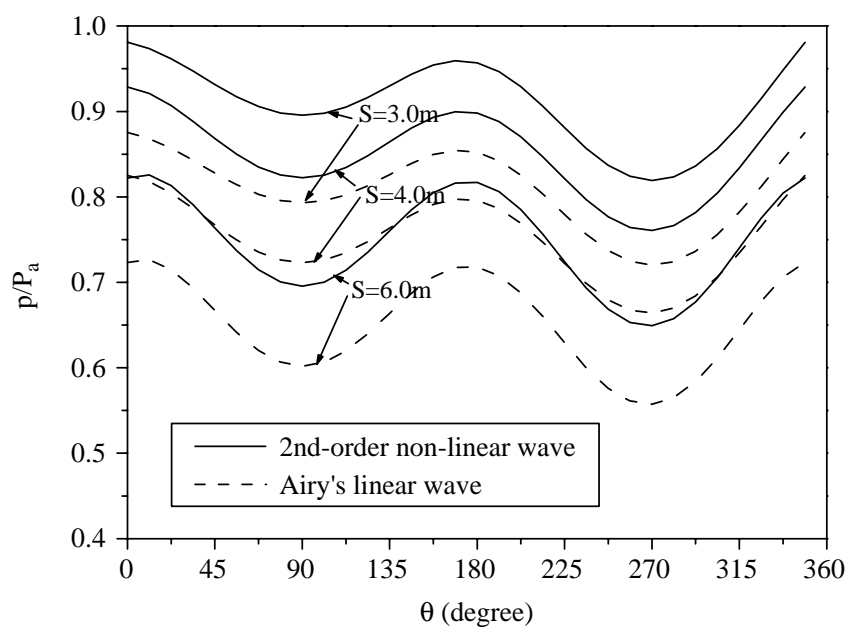

Fig. 12. Distribution of wave-induced excess pore pressure along trenched pipeline for various trench depths. 
$\alpha=45^{\circ}$ and $B=3.0 \mathrm{~m}$ as an example. Fig. 12 shows the distribution of excess pore pressure along pipeline for various trench depths. With the increase of $S$, the waveinduced excess pore pressure decreases greatly, which implies that a larger trench depth could enhance the potential of soil instability around pipeline. The influences of trench depth are much more obvious than those of slope angle of trench. Fig. 12 also indicates that the influences of wave non-linearity are comparable with those of trench depth. Therefore, the non-linear wave loading should be taken into account when designing the trench depth of pipeline.

\section{Conclusions}

A two-dimensional finite element model is developed to investigate the non-linear wave-induced transient response of soil around a trenched pipeline, which is verified with flume test results by Turcotte et al. (1984) and Sudhan et al. (2002). The effects of wave non-linearity and backfill soil properties on the distribution of excess pore pressure are examined parametrically. Based on the numerical results, the following conclusions can be drawn:

(1) The bottom pressure fluctuations induced by non-linear waves have a direct and continuous influence on changes in pore pressure along pipeline under various phases of a progressive wave. The effects of wave non-linearity on excessive pore pressures around trenched pipeline get more obvious when increasing the wave steepness in shallow water zones.

(2) Both the shear modulus $(G)$ and permeability coefficient $(k)$ of backfill soil have much influence on soil responses around the trenched pipeline. With the increase of $G$, the wave-induced excess pore pressures $\left(p / P_{\mathrm{a}}\right)$ around pipeline decrease. The distribution of $p / P_{\mathrm{a}}$ is also affected by the permeability of backfill. Coarser the backfill sand, higher the excess pore pressures at the zone near the seabed mudline. However, the values of $p / P_{\mathrm{a}}$ beneath the pipeline have a trend of getting lower in the coarser backfill.

(3) The geometry profile of trenches also has influence on the wave-induced excess pore pressures within soil around pipeline. With the increase of trench depth, non-linear wave-induced excess pore pressures along pipeline get much lower. The influences of slope angle of trench are not as much as those of trench depth. It is noted that the effect of wave non-linearity becomes more pronounced and comparable with that of trench depth, especially at high wave steepness in shallow water.

\section{Acknowledgements}

Financial support by 'Tenth Five-year Plan' of Chinese Academy of Sciences (Project No. KJCX2-SW-L03) is greatly appreciated. The authors also thank Dr Dong-Sheng Jeng at the University of Sydney for his valuable discussions. 


\section{Appendix A. List of coefficient matrices}

The coefficients $B_{i}(i=1-5)$ in Eqs. (19), (20) and (28) are listed as follows:

$$
\begin{aligned}
& B_{i}=\left[\begin{array}{llll}
b_{i 1} & b_{i 2} & \cdots & b_{i n_{\mathrm{e}}}
\end{array}\right], \\
& b_{1 i}=\left[\begin{array}{cc}
\frac{\partial N_{i}}{\partial x} & 0 \\
0 & \frac{\partial N_{i}}{\partial x} \\
\frac{\partial N_{i}}{\partial z} & 0 \\
0 & \frac{\partial N_{i}}{\partial z}
\end{array}\right]=b_{3 i}^{\mathrm{T}}, \\
& b_{2 i}=\left[\begin{array}{cc}
N_{i} & 0 \\
0 & N_{i}
\end{array}\right] \text {, }
\end{aligned}
$$

$$
b_{4 i}=\left[\begin{array}{cccc}
\frac{\partial N_{i}}{\partial x} & 0 & 0 & 0 \\
0 & \frac{\partial N_{i}}{\partial x} & 0 & 0 \\
0 & 0 & \frac{\partial N_{i}}{\partial z} & 0 \\
0 & 0 & 0 & \frac{\partial N_{i}}{\partial z} \\
\frac{\partial N_{i}}{\partial z} & 0 & \frac{\partial N_{i}}{\partial x} & 0 \\
0 & \frac{\partial N_{i}}{\partial z} & 0 & \frac{\partial N_{i}}{\partial x}
\end{array}\right] \text {, }
$$

$$
b_{5 i}=N_{i}\left[\begin{array}{cccc}
1 & 0 & 0 & 0 \\
0 & 1 & 0 & 0 \\
0 & 0 & 1 & 0 \\
0 & 0 & 0 & 1
\end{array}\right] \text {. }
$$


The coefficient matrices $D_{i}$ in (19), (20) and (28) are given as

$$
\begin{aligned}
& D_{1}=\frac{\gamma_{\mathrm{w}}}{k}\left[\begin{array}{llll}
1 & 0 & 0 & 0 \\
0 & 1 & 0 & 0 \\
0 & 0 & 1 & 0 \\
0 & 0 & 0 & 1
\end{array}\right] \text {, } \\
& D_{2}=\frac{n \omega}{K^{\prime}}\left[\begin{array}{cc}
0 & -1 \\
1 & 0
\end{array}\right] \text {, } \\
& D_{3}=\omega\left[\begin{array}{cc}
-1 & 0 \\
0 & 1
\end{array}\right] \text {, } \\
& D_{4}=\frac{2 G}{1-2 \mu}\left[\begin{array}{cccccc}
1-\mu & 0 & \mu & 0 & 0 & 0 \\
0 & 1-\mu & 0 & \mu & 0 & 0 \\
0 & 0 & 1-\mu & 0 & 0 & 0 \\
0 & 0 & 0 & 1-\mu & 0 & 0 \\
0 & 0 & 0 & 0 & \frac{1-2 \mu}{2} & 0 \\
0 & 0 & 0 & 0 & 0 & \frac{1-2 \mu}{2}
\end{array}\right] \text {, } \\
& D_{5}=\frac{2 G_{\mathrm{p}}}{1-\nu}\left[\begin{array}{cccccc}
1-\nu & 0 & \nu & 0 & 0 & 0 \\
0 & 1-\nu & 0 & \nu & 0 & 0 \\
0 & 0 & 1-\nu & 0 & 0 & 0 \\
0 & 0 & 0 & 1-\nu & 0 & 0 \\
0 & 0 & 0 & 0 & \frac{1-2 \nu}{2} & 0 \\
0 & 0 & 0 & 0 & 0 & \frac{1-2 \nu}{2}
\end{array}\right] .
\end{aligned}
$$




\section{References}

Biot, M.A., 1941. General theory of three-dimensional consolidation. Journal of Applied Physics 12, 115-129.

Cheng, A.H.D., Liu, P.L.F., 1986. Seepage force on a pipeline buried a poroelastic seabed under wave loadings. Applied Ocean Research 8, 22-32.

Clukey, E.C., Vermersch, J.A., Koch, S.P., Lamb, W.C., 1989. Natural densification by wave action of sand surrounding a buried offshore pipeline, Proceedings of the 21st Annual Offshore Technology Conference, Houston, TX 1989 pp. 291-300.

Gatmiri, B., 1990. A simplified finite element analysis of wave-induced effective stresses and pore pressures in permeable sea beds. Géotechnique 40, 15-30.

Jeng, D.-S., 1997. Soil response in cross-anisotropic seabed due to standing waves. Journal of Geotechnical and Geoenvironmental Engineering, ASCE 123 (1), 9-19.

Jeng, D.-S., 2001. Numerical modeling for wave-seabed-pipe interaction in a non-homogeneous porous seabed. Soil Dynamics and Earthquake Engineering 21, 699-712.

Jeng, D.-S., 2003. Wave-induced seafloor dynamics. Applied Mechanics Review 56 (4), 407-429.

Jeng, D.-S., Lin, Y.S., 1996. Finite element modelling for water waves-seabed interaction. Soil Dynamics and Earthquake Engineering 15, 283-300.

Laitone, E.V., 1962. Limiting conditions for Cnoidal and Stokes waves. Journal of Geophysics Research 67, 1555-1564.

MacDougal, W.G., Davidson, S.H., Monkmeyer, P.L., Sollitt, C.K., 1988. Wave-induced forces on buried pipelines. Journal of Waterway, Port, Coastal and Ocean Engineering, ASCE 14, 220-236.

MacPherson, H., 1978. Wave forces on pipelines buried in permeable seabed. Journal of Waterway, Port, Coastal and Ocean Division, ASCE 104, 407-419.

Madsen, O.S., 1978. Wave-induced pore pressures and effective stresses in a porous bed. Géotechnique 28, 377393.

Magda, W., 1997. Wave-induced uplift force acting on a submarine buried pipeline in a compressible seabed. Ocean Engineering 24, 551-576.

Mei, C.C., Foda, M.A., 1981. Wave-induced stresses around a pipe laid on a poro-elastic sea bed. Géotechnique 31, 509-517.

Spierenburg, S.E.J., 1986. Wave-induced pore pressures around submarine pipelines. Coastal Engineering 10, $33-48$.

Sudhan, C.M., Sundar, V., Rao, S.N., 2002. Wave induced forces around buried pipeline. Ocean Engineering 29, 533-544.

Thomas, S.D., 1989. A finite element model for the analysis of wave induced stresses, displacements and pore pressures in an unsaturated seabed. I: Theory. Computers and Geotechnics 8, 1-38.

Turcotte, B.R., Liu, P.L.F., Kulhawy, F.H., 1984. Laboratory evaluation of wave tank parameters for wavesediment interaction. Joseph H. Defree Hydraulic Laboratory Report 84-1, School of Civil and Environmental Engineering, Cornell University.

Yamamoto, T., Koning, H.L., Sellmeiher, H., Hijum, E.V., 1978. On the response of a poro-elastic bed to water waves. Journal of Fluid Mechanics 87, 193-206.

Zen, K., Yamazaki, H., 1990. Mechanism of wave-induced liquefaction and densification in seabed. Soils and Foundations 30, 90-104.

Zienkiewicz, O.C., Scott, F.C., 1972. On the principle of repeatability and its application in analysis of turbine and pump impellers. International Journal for Numerical Methods in Engineering 9, 445-452.

Zienkiewicz, O.C., Taylor, R.L., 1989. The Finite Element Method, fourth ed. McGraw-Hill, London. 